\title{
Tendencias internacionales en materia de política educativa desde el contexto colombiano
}

\author{
International trends in educational \\ policy concerns from the Colombian \\ context
}

\author{
Derly Janeth Forero Hernández
}

Recepción:03/04/2020 • Aprobación:27/04/2020 • Publicación: 12/08/2020

Para citar este artículo

Forero Hernández, D. J. (2020). Tendencias internacionales en materia de política educativa desde el contexto colombiano. Dos mil tres mil, 22, e229.

https://doi.org/10.35707/dostresmil/22229

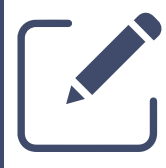

\section{(c) (1) $\circledast \Theta$}

"Políticas Educativas y Entorno Educativo, Docente de Aula, Secretaria de Educación Departamental -Tolima. ORCID: 0000-00020472-7720. Correo electrónico: derlyforero7@gmail.com 
Resumen. El objetivo de este artículo es evidenciar que actualmente para muchos de los ciudadanos la educación es solo un derecho; para afirmar esto, basta con observar las políticas públicas de cada gobierno y, aún más, mirar desde los organismos internacionales uno de sus objetivos de desarrollo sostenible. En el cuarto objetivo encontramos una educación de calidad, la cual comprende gratuidad, cero analfabetización, educación inclusiva, equitativa, para promover la oportunidad y formación por competencias. Sin embargo, acceder a ello, se convierte en un problema económico y social, de aquí que la educación deja de ser un derecho.

Abstract. The aim of this article is to show that nowadays education is only a right for many of the citizens; to affirm this, it is enough to observe the public policies of each government and, even more, to take a look from international organizations at one of its objectives of sustainable development. In the fourth objective we find quality education, which includes to have no charge, zero illiteracy, inclusive and equitable education, in order to promote opportunity and training through skills. However, having access to it becomes an economic and a social problem; hence, education is no longer a right.

Palabras claves

Globalización, sistema y políticas educativas, cambios tecnológicos, desarrollo de competencias.

Keywords

Globalization, educational system and policies, technological changes, development of competences. 


\section{Introducción}

Para los organismos internacionales, los temas prioritarios en educación hacen parte de la agenda, pero con fines económicos. Esto pone a la educación en un plano en el que se aleja de un derecho y se convierte en un servicio.

La realización del siguiente artículo, por una parte, permite ahondar en el conocimiento y posibles temas de investigación desde los acuerdos internacionales y la educación, así como los impactos en el tiempo en la calidad educativa; por otra parte, Colombia ha tenido una positiva transformación e importantes avances en materia de desarrollo, crecimiento económico, reducción de la pobreza y generación de empleo; sin embargo, aún enfrenta grandes desafíos en los cuales la cooperación internacional es una herramienta clave para superarlos, como lo es la construcción de paz (Rettberg et al., 2018). Un aspecto positivo, es la oportunidad que se les brinda a los estudiantes para poder intercambiar, con otras instituciones de diferentes países, experiencias y conocimientos propios. Además, con algunos de estos convenios se pretende acabar con el analfabetismo y permitir la cobertura al $100 \%$ en educación, al atender las necesidades; para ello, existen apoyos por parte de personas idóneas que trabajan en el ámbito educativo, exigiendo calidad en los programas.

Por lo anterior, el presente documento pretende analizar y explicar las tendencias macro (sociales, políticas y económicas), además de reconocer sus efectos en los sistemas educativos latinoamericanos.

\section{Los desafíos de la globalización en los sistemas educativos latinoamericanos}

El tema de la globalización es realmente complejo, puesto que existen puntos de vista y orientaciones distintas según el tipo de autor que refiere al concepto y a su análisis frente a los sistemas educativos de Latinoamérica, pero en los autores que brindan su conceptualización existe una relación cuando lo definen desde términos económicos, neoliberal o capitalista, lo cual es evidente en lo observado en las políticas públicas.

Es así que se puede definir la globalización como el conjunto de procesos relacionados a factores que afectan e influyen en el desarrollo de los países, asimismo lo afirma López Rupérez (2001), citado por Sánchez y Rodríguez (2011), que se puede identificar la globalización como:

Un conjunto de procesos, principalmente de carácter económico, que, a través de las interacciones, interconexiones e interdependencias existentes entre los diversos países y de la intensificación, desarrollo y extensión de esas redes globales, produce que ciertos hechos, acciones y decisiones ocurridos en un lugar concreto del globo y que antes sólo repercutían localmente, ahora lo hagan de forma global. (Sánchez \& Rodríguez, 2011, p. 1).

Por esta razón, la globalización influye significativamente en la educación, ya que para su funcionamiento depende del sistema educativo desde la política pública. Así, dentro de sus 
políticas se encontrará la educación para la formación laboral, competitiva, entre otros, lo cual permite un mejoramiento en el desarrollo social y económico en el sector productivo.

En los desafíos que presenta la globalización frente a la educación, se encuentra la formación de seres humanos para el sector productivo, ciudadanos funcionales al sistema de producción capitalista, de ahí el interés por cambiar el currículo en formación laboral, áreas financieras, contenidos referidos a la competitividad a través de una reforma educativa, en la que la educación se privatice y permita lograr cambios representativos y de conveniencia para las políticas internacionales relacionadas al capitalismo.

De este modo, como docentes queda el sinsabor del fin principal de la educación y se convierte en un objeto de la globalización, del cual no hay salida y solo se debe seguir la ruta establecida por esta, al entender claramente los factores que afectarán significativamente en algunos sectores y ampliamente mejorarán en el sector económico y productivo.

\section{Visiones sobre desarrollo y educación}

En el interés en la globalización por el desarrollo económico, la educación pasa a ser el principal actor para lograrlo, ya que este es el espacio en el que se adquiere conocimiento, pensamiento crítico y preparación para la vida. Por lo anterior, Mejía (2010) afirma que tanto la tecnología como el conocimiento son los recursos principales para lograr en los seres humanos, en su educación, formación en investigación, información, comunicación y actualización en tecnología y conocimiento, y así convertirse en los mayores bienes económicos de la sociedad.

Por consiguiente, a partir de las políticas públicas se establece la necesidad de la formación de humanos basada en competencias, emprendimiento, habilidades laborales, desarrollo técnico, al tener en cuenta que en uno de los objetivos principales de la globalización y en las organizaciones internacionales, se evidencia el interés por el desarrollo económico desde el sector productivo, a su vez la educación debe ir enlazada con el interés de la formación con dicho fin. Para Saxe (1999), citado por Avendaño y Guacaneme (2016):

La pasividad y el conformismo, ya que se asume que las fuerzas del mercado global poseen las capacidades extraordinarias para determinar y limitar las opciones y las políticas, como si la dinámica interna y las relaciones de clase hayan cesado de operar. (Avendaño \& Guacaneme, 2016, p. 199).

Por esta razón, las políticas educativas deben adecuarse al modelo capitalista, lo cual lleva a reorientarse a nuevos discursos al desplazarse a un interés común por parte del mercado, ilustrándose como el lugar donde no solo se adquiere conocimientos, sino como una empresa sometida a estándares de calidad, con parámetros definidos en eficiencia y productividad; para el caso de Colombia, la educación no formal, en la que los ciudadanos se forman para ser competentes laboralmente. Como ejemplo, el Sistema Nacional de Aprendizaje (sENA), encargado de la formación técnica y tecnológica, preparada para el sector productivo al cumplir la función que las políticas públicas necesitan, para lograr el desarrollo económico del país. 


\section{Impacto de los cambios tecnológicos}

Dentro de los cambios observados en el sistema educativo encontramos la utilización y actualización referida a la tecnología, la información y la comunicación; así, las políticas educativas en la integración de las tecnologías en las instituciones educativas han intentado mejorar en infraestructura, equipamiento y principalmente en el acceso a la información en los centros educativos, a través de inversión tecnológica y programas que permitan la formación de seres humanos competentes.

Aunado a lo anterior, en las últimas décadas, las políticas nacionales y regionales han implementado desde las políticas educativas la integración de las Tecnologías de la Información y la Comunicación (TIC) en el sistema educativo, de las cuales, como lo afirma Valverde Berrocoso et al., (2010): "Algunas de ellas son políticas 'estratégicas' que tratan de proporcionar un conjunto de metas y una visión acerca del papel de las tecnologías en los procesos educativos y sus potenciales beneficios" (p. 101). A lo anterior, el autor agrega que "las políticas educativas cuando han sido bien definidas pueden motivar, fomentar la innovación y coordinar los esfuerzos de los diferentes agentes de la comunidad educativa" (p. 101).

Por lo anterior, las tecnológicas se han convertido en el apoyo para el desarrollo económico al generar un cambio socio cultural; asimismo, las políticas educativas con la finalidad de mejorar y cambiar las prácticas de enseñanza-aprendizaje y así lograr una transformación social en los sistemas educativos del país. Para ello, se han adoptado cuatro aplicaciones de las tecnologías a la educación por parte de Kozma (2005), citado por Valverde Berrocoso et al.:

(a) Las TIC son utilizadas para facilitar el acceso a la educación. Este enfoque mejora la educación incrementando las posibilidades de formación, pero no implica necesariamente un cambio educativo fundamental;

(b) las TIC se orientan hacia el aprendizaje. Gracias a la adquisición de competencias tecnológicas, los estudiantes están mejor preparados para su inserción laboral;

(c) las TIC pueden ser utilizadas para mejorar la comprensión de los conocimientos por parte de los alumnos, pueden contribuir a la calidad de la educación y, en consecuencia, pueden aumentar el impacto de la educación sobre la economía, y

(d) la creación y compartir el conocimiento, así como la innovación tecnológica, pueden contribuir a la transformación del sistema educativo y el sostenimiento del desarrollo económico y social. (Valverde Berrocoso et al., 2010, pp. 101-102).

No obstante, para lograr lo anterior, se debe contar con docentes con formación en el uso de las tecnologías, asimismo incluir en sus prácticas pedagógicas el desarrollo de un aprendizaje significativo desde las tecnologías como herramienta para el desarrollo de actividades y de adquisición de conocimiento, y así comprometer al profesorado en el desarrollo de un plan TIC que permita la oportunidad de reflexionar sobre su particular uso educativo, al encontrar el significado subjetivo de cómo y por qué utilizan las Tic en sus prácticas docentes (Somekh, 2008). 
Para lo anterior, se requiere generar inversión en investigación científica y tecnológica, en la formación y actualización de los docentes en el uso adecuado y correcto de las tecnologías; así, si de las políticas públicas parte el interés por invertir económicamente en la educación, se verá reflejado la trasformación del sistema educativo y el desarrollo capitalista a partir de la tecnología, que es en sí el boom mundial.

\section{Generación de conocimiento y desarrollo de competencias}

Al continuar con el interés por la globalización en diferentes actividades de la sociedad, se ha observado el creciente desarrollo de la tecnología en la educación, lo cual permite mejorar asertivamente la economía del país, y a su vez los cambios culturales que generan articulación de diferentes culturas con un propósito en común: la competencia; por ello es fundamental la necesidad de actuar en un mundo globalizado, con conocimiento de las economías internacionales, la influencia de organismos internacionales, y el pensamiento crítico frente al desarrollo del país desde una mirada capitalista y tecnológica.

Por consiguiente, la educación cambia significativamente la función del docente en formar ciudadanos competentes en lo laboral, eficiencia, y capacidad de adaptación en el sector laboral; se exigen personas altamente capacitadas con habilidades para decidir qué hacer, cómo hacerlo, y definir lo correcto de lo incorrecto para la empresa, y de esta forma saber actuar frente a una situación determinada. Por lo tanto, el docente debe generar propuestas de enseñanza para desarrollar competencias necesarias y lograr de esta manera un buen desempeño en lo laboral y personal.

A su vez, la educación debe cambiar las prácticas pedagógicas pensando en la formación por competencias, al recordar que Colombia desde las políticas educativas incluye el modelo por competencias dentro de las instituciones educativas en su Proyecto Educativo Institucional (PEI), y tener en cuenta que se viene encaminando la formación de esta manera, con el fin de fortalecer el desarrollo productivo del país a partir de ciudadanos capaces de cumplir con la funciones encargadas. De este modo, la educación forma ciudadanos competentes para laborar de manera eficaz. Aunando a lo anterior, Nagles afirma:

El escenario actual es altamente competitivo, se requiere desarrollar la capacidad creativa y la habilidad para generar soluciones novedosas e innovadoras a los problemas y dificultades, así como, a las demandas de los clientes [...] el desarrollo de competencias se logra en un escenario en el que interactúan, en forma dinámica, cuatro elementos fundamentales: personas, organizaciones, estrategias y conocimiento de manera racional; es decir, se alcanza una mayor efectividad con un menor esfuerzo. (Nagles, 2005, pp. 100-101).

Por esta razón, para lograr desarrollar las competencias necesarias para ser "útil" en la vida laboral de manera efectiva y productiva, se requiere cambiar la forma de pensar, actuar y sentir; es decir, tener una perspectiva abierta a la adaptación, reflexión, y crítica para mejorar y desarrollar habilidades comunicativas y de resiliencia; esto implica generar estrategias que 
motiven y gestionen a los profesionales y a los trabajadores para contribuir a la transformación de la sociedad.

\section{Prioridades de los sistemas educativos en Latinoamérica}

En las organizaciones internacionales, tanto la UNESCO como la OCDE tienen como objetivo principal el mejoramiento de la calidad educativa; para el caso de América Latina y el Caribe, la situación educativa está estrechamente ligada a factores tanto externos como internos del sistema educativo que no permite ver con claridad las metas para lograr la excelencia educativa, por ello la UNESCO (2013) estructura las prioridades en los sistemas educativos de la siguiente manera:

- Inversión pública en educación en América Latina y el Caribe.

- El cuidado y la educación de la primera infancia.

- Enseñanza primaria: acceso e inclusión.

- El desafío de la calidad de la educación: logros de aprendizaje y aseguramiento de la calidad, docentes y calidad de la educación, clima escolar y su relación con la calidad de la educación, educación para la ciudadanía y calidad de la educación y tecnologías de la información y comunicación.

- Educación superior: acceso.

- Equidad de género.

- Educación intercultural bilingüe: diversidad.

- Alfabetización de adultos y aprendizaje a lo largo de la vida.

Aunado a lo anterior, los objetivos del Foro Marco de Acción de Dakar (2000) han permitido evidenciar avances, al adicionar dentro de las políticas públicas como metas, para la calidad de la educación, los objetivos anteriormente nombrados; se encuentra la iniciativa de la UNESCO enunciada como la Educación Para Todos (EPT), que busca brindar educación básica de calidad a todos los niños, jóvenes y adultos. Asimismo, se objetiva, según el Marco de Acción de Dakar (2000), para el sistema educativo:

- La educación y cuidado de la primera infancia.

- La educación primaria universal.

- El aprendizaje de jóvenes y adultos.

- La alfabetización.

- La paridad de género.

- La calidad de la educación

\section{La dimensión internacional de la educación}

La cooperación internacional en la educación en Colombia ha adquirido cada vez más gran importancia, al adoptar formas variadas y sofisticadas en su participación. Estas incluyen: 
La internacionalización de los programas académicos que ofrecen las instituciones, la creación de dobles titulaciones en colaboración con instituciones extranjeras, la apertura en el extranjero de facultades dependientes de las instituciones, la convalidación internacional de títulos y competencias, la acreditación de las instituciones de educación superior y de los programas que llevan a cabo por organismos extranjeros, la creación de centros de idiomas y el establecimiento del requisito del bilingüismo o multilingüismo para la obtención de títulos de educación superior, el aumento de la participación de investigadores en equipos internacionales con colegas de otros países, la aceptación de los cursos a distancia ofrecidos por instituciones extranjeras en el marco del plan de estudios ordinario de los estudiantes nacionales, la participación en consorcios internacionales, y el deseo de las instituciones y de los gobiernos de formar parte del ranking mundial de universidades. (OCDEBanco Mundial, 2012, p. 228).

Desde la política educativa actual en Colombia, al igual que en los países latinoamericanos, se cumple con las políticas educativas al tener en cuenta lo dicho por la Organización de Cooperación y el Desarrollo Económico (OCDE); asimismo, el Banco Mundial, en el que es evidente observar el interés principal en reflejar datos estadísticos referente a la calidad educativa, sin tener en cuenta las necesidades actuales de los países y de por sí, las diversas culturas encontradas en cada uno de estos; así lo afirma la oCDE referido a uno de los temas de gran interés en optimizar la calidad educativa: "La jornada única es una oportunidad para mejorar la infraestructura y mejorar la calidad y la igualdad, pero deben abordarse retos en cuanto a su implementación” (Radinger et al., 2018, p. 18).

Frente a lo anterior, es evidente el interés por incrementar la calidad educativa, por esto la política actual por cumplir lo estipulado por la OCDE solo se convierte en una elección por parte de los políticos en adoptar aquellas medidas que conduzcan a mejorar y lograr los resultados en la calidad educativa; sin embargo, para alcanzarlos no se trata solo de estipular los cambios que debe hacer cada entidad educativa, sino que se necesita de apoyo económico para un desarrollo sostenible, en cuanto a los factores externos que influyen en mejorar la calidad educativa; ejemplo de ello es la infraestructura, el perfil de los profesores, los contextos, las culturas, entre otros.

En Colombia, la educación es un derecho fundamental de todo ciudadano, según el artículo 67 de la Constitución Política de 1991, el Estado debe velar por la calidad de la educación, garantizar la cobertura y asegurar a los menores las condiciones necesarias para el acceso y la permanencia en la escuela. Lo anterior implica que el núcleo esencial del derecho fundamental de la educación incluye los siguientes elementos básicos:

- El derecho a la disponibilidad del servicio educativo.

- El derecho que garantiza el acceso al mismo.

- El derecho a que el servicio educativo sea de calidad.

- El derecho a que se garantice la permanencia de los niños, las niñas y los jóvenes en el mismo.

Por lo anterior, al tener en cuenta los objetivos dispuestos por las organizaciones internacionales en cuanto a la calidad educativa, las políticas públicas deben ir encaminadas de igual 
manera a los mismos objetivos y así, en un trabajo colectivo en la política educativa; de esta manera, se proyectarán resultados para el mejoramiento de la calidad educativa no solo en la adquisición de conocimiento sino en la formación humana, laboral, competente, entre otros.

\section{Reconocimiento de las necesidades regionales}

En Colombia es evidente observar el sistema educativo no esperado, por varios factores que influyen para no obtener los objetivos planteados, algunos de ellos son: la falta de inversión para infraestructura, alimentación escolar, transporte escolar, actualización en la formación de docentes, vías adecuadas, educación rural, inclusión, entre otros; el Gobierno prefiere más la inversión en el sector económico y productivo que en la política educativa; por esta razón existen necesidades regionales, frente a las cuales se deben buscar prontas soluciones para avanzar en la calidad educativa. Sin embargo, a pesar de los progresos, aún queda mucho por abarcar para afrontar algunos desafíos, como: aumentar las matrículas y fomentar la equidad, mejorar la calidad y la pertinencia, así como lograr que la gestión y las finanzas sean más apropiadas.

Entre otros aspectos, el Gobierno necesita aumentar la participación en la educación postsecundaria, al mejorar los préstamos y los sistemas de becas e incrementar el número de cupos de la manera más equitativa posible en todo el país. En muchos casos, la internacionalización se limita a la movilidad estudiantil y a la firma de acuerdos internacionales por parte de los responsables institucionales. Por consiguiente, entre las necesidades evidentes en Colombia que afectan significativamente la calidad educativa, encontramos:

- Cobertura al $100 \%$.

- Transporte escolar.

- Alimentación escolar.

- Inclusión de niños con necesidades educativas especiales.

- Desplazamiento por el conflicto armado.

- Intereses personales en la política pública.

- Formación profesional docente.

\section{Conclusiones}

Tanto los enfoques como las características de las políticas públicas están estrechamente relacionados al tipo de sociedad que se quiere y las necesidades de esta. Por esta razón, se deben analizar los programas internacionales que trabajan en conjunto para obtener la calidad educativa esperada. Para ello, las tecnologías de la información y comunicación, y los programas realizados por el Gobierno colombiano que permitan intentar dar solución y lograr los objetivos propuestos por las organizaciones, se deberán analizar y explicar las tendencias macro (sociales, políticas y económicas), además de reconocer sus efectos en los sistemas educativos latinoamericanos, y así observar el alcance de las organizaciones y efectuar cambios que mejoren el desarrollo económico del país. 
La educación se convierte en el ente principal para fortalecer el desarrollo económico, en cuanto a la formación profesional, humana, en principios, con igualdad de condiciones y metas planteadas con coherencia e interés público.

\section{Referencias}

Avendaño Castro, W. R., \& Guacaneme Pineda, R. E. (2016). Educación y globalización: Una visión crítica. Civilizar Ciencias Sociales y Humanas, 16(30), 191-206. https://doi.org/10.22518/16578953.543

Foro Marco de Acción de Dakar (2000). Educación para todos: Cumplir nuestros compromisos comunes. (Con los seis Marcos de Acción Regionales). Dakar, Senegal, 26 al 28 de abril de 2000. Recuperado de https://unesdoc.unesco.org/ark:/48223/pf0000121147_spa

Mejía, M. R. (2010). Las pedagogías críticas en tiempos de capitalismo cognitivo. Cartografiando las resistencias en educación. Revista Aletheia, Revista de Desarrollo Humano, Educativo y Social Contemporáneo, 2(2), 58-101. Recuperado de https://aletheia.cinde.org.co/index.php/ALETHEIA/article/view/27

Nagles García, N. (2005). El desarrollo de competencias. Revista Escuela de Administración de Negocios, (54), 99-114. Recuperado de https://journal.universidadean.edu.co/index.php/Revista/article/ view/335/326

ocDe-Banco Mundial (2012). Evaluaciones de Políticas Nacionales de Educación: La Educación Superior en Colombia 2012. Recuperado de https://www.oecd.org/education/skills-beyond-school/Evaluaciones\%20de\%20pol\%C3\%ADticas\%20nacionales\%20de\%20Educaci\%C3\%B3n\%20-\%20La\%20 Educaci\%C3\%B3n\%20superior\%20en\%20Colombia.pdf

Radinger, T., Echazarra, A., Guerrero, G., \& Valenzuela J. P. (2018). oECD Revisión de recursos escolares Colombia. Recuperado de https://www.oecd.org/education/school/OECD-Reviews-School-Resources-Summary-Colombia-Spanish.pdf

Rettberg, A., Medina, D., \& Pimienta, L. (2018). Estrategias del empresariado colombiano en la construcción de Paz (2017-2018) (Colombian Business Strategies in Peacebuilding (2017-2018)). Recuperado de https://papers.ssrn.com/sol3/papers.cfm?abstract_id=3242735

Sánchez Delgado, P., \& Rodríguez Miguel, J. C. (2011). Globalización y educación: Repercusiones del fenómeno en los estudiantes y alternativas frente al mismo. Revista Iberoamericana de Educación, 54(5): 1-12. https://doi.org/10.35362/rie5451653

Somekh, B. (2008). Factors affecting teachers' pedagogical adoption of ICT. En J. Voogt \& G. Knezek (Eds.), International Handbook of Information Technology in Primary and Secondary Education (pp. 449-460). New York: Springer.

unesco (2013). Situación educativa de América Latina y el Caribe: Hacia la educación de calidad para todos al 2015. Oficina Regional de Educación para América Latina y el Caribe. Recuperado de http:// www.unesco.org/new/fileadmin/MULTIMEDIA/FIELD/Santiago/images/SITIED-espanol.pdf

Valverde Berrocoso, J., Garrido Arroyo, M. D. C., \& Sosa Díaz, M. J. (2010). Políticas educativas para la integración de las Tic en Extremadura y sus efectos sobre la innovación didáctica y el proceso enseñanza-aprendizaje: La percepción del profesorado. Revista de Educación, 352, 99-124. Recuperado de http://www.revistaeducacion.educacion.es/re352/re352_05.pdf 